\title{
OPEN Association between red blood cell distribution width and long-term mortality in acute respiratory failure patients
}

\author{
Wei Zhang ${ }^{1 凶}$, Yadan Wang ${ }^{2}$, Jun Wang ${ }^{1} \&$ Shaochun Wang ${ }^{1}$
}

The red cell distribution width (RDW) has been reported to be positively correlated with short-term mortality of pulmonary disease in adults. However, it is not clear whether RDW was associated with the long-term prognosis for acute respiratory failure (ARF). Thus, an analysis was conducted to evaluate the association between RDW and 3-year mortality of patients by the Cox regression analysis, generalized additives models, subgroup analysis and Kaplan-Meier analysis. A total of 2999 patients who were first admitted to hospital with ARF were extracted from the Medical Information Mart for Intensive Care III database (MIMIC-III). The Cox regression analysis showed that the high RDW was associated with 3-year mortality (HR 1.10, 95\% CI 1.07, 1.12, $\mathrm{P}<0.0001)$ after adjusting for age, gender, ethnicity and even co-morbid conditions. The ROC curve illustrated the AUC of RDW was 0.651 $(95 \% \mathrm{Cl} 0.631,0.670)$ for prediction of 3-year mortality. Therefore, there is an association between the RDW and survival time of 3 years follow-up, particularly a high RDW on admission was associated with an increased risk of long-term mortality in patients with ARF. RDW may provide an alternative indicator to predict the prognosis and disease progression and more it is easy to get.

Acute respiratory failure (ARF) is one of the most common complications in critically ill patients admitted to the intensive care unit (ICU) and usually leads to high mortality ${ }^{1}$. Even with mechanical ventilation and extracorporeal membrane oxygenation (ECMO), approximately $36 \%$ of patients died consequently during hospitalization ${ }^{2}$. Although there are some scoring systems used to predict the clinical outcomes of patients in ICU, no one is specifically available for patients with AFR. Several studies have demonstrated that hypoproteinemia, malnutrition, predecease functional status, and Acute Physiology and Chronic Health Evaluation II (APACHE II) score ${ }^{3,4}$ are related to the need for mechanical ventilation in ARF, but most of these studies were conducted in patients with acute exacerbation of chronic obstructive pulmonary disease (AECOPD) who made up only a small proportion of patients with ARF. Delayed recognition of clinical deterioration in AFR is common, which leads to poor clinical outcomes and increased in-hospital mortality ${ }^{5}$. Therefore, it is necessary to find useful indicators to predict the clinical outcomes of ARF.

Red blood cell distribution width (RDW) is a parameter of red blood cell volume heterogeneity obtained by a standard complete blood count ${ }^{6}$. It is commonly used to identify different types of anemia and reactive bone marrow states and it is also a simple, widely used, and inexpensive parameter. In recent years, some clinical studies have shown that there is a correlation between RDW and some acute diseases, such as brain infarction, sepsis, infective endocarditis and diabetic ketoacidosis ${ }^{7-11}$. In addition, several studies have reported the predictive effect of RDW on lung disease ${ }^{12-16}$, while the predictive effect of RDW on acute respiratory failure has not been reported. Therefore, our objective is to study whether RDW is useful to predict clinical outcomes of patients with ARF.

\section{Results}

Characteristics of patients. There were 2999 patients with ARF included in the study, with 1654 (55.15\%) patients died within 3 years. We divided all the patients into three groups, according to the tertiles of RDW: the low group $(\mathrm{RDW}<14.1 \%)$, the middle group $(14.1 \% \leq \mathrm{RDW}<15.7 \%)$ and the high group $(\mathrm{RDW} \geq 15.7 \%)$. Patients with high RDW were more likely to have high APS III score, OASIS score and more comorbidities. They 


\begin{tabular}{|c|c|c|c|c|c|}
\hline & \multirow[b]{3}{*}{ All subjects } & \multicolumn{3}{|l|}{ RDW (\%) } & \multirow[b]{3}{*}{ P-value } \\
\hline & & $<14.1$ & $\geq 14.1<15.7$ & $\geq 15.7$ & \\
\hline & & $\mathrm{N}=947$ & $\mathrm{~N}=1049$ & $N=1003$ & \\
\hline Age (years) & $64.88 \pm 16.57$ & $62.12 \pm 18.34$ & $66.28 \pm 15.90$ & $66.02 \pm 15.14$ & $<0.001$ \\
\hline Female & $1348(44.95 \%)$ & $412(43.51 \%)$ & $456(43.47 \%)$ & $480(47.86 \%)$ & 0.076 \\
\hline \multicolumn{6}{|l|}{ Ethnicity } \\
\hline Caucasian & $2072(69.09 \%)$ & $649(68.53 \%)$ & $747(71.21 \%)$ & $676(67.40 \%)$ & 0.005 \\
\hline Black & $208(6.94 \%)$ & $61(6.44 \%)$ & $52(4.96 \%)$ & $95(9.47 \%)$ & \\
\hline Asian & $71(2.37 \%)$ & $20(2.11 \%)$ & $25(2.38 \%)$ & $26(2.59 \%)$ & \\
\hline Others & $648(21.61 \%)$ & $217(22.91 \%)$ & $225(21.45 \%)$ & $206(20.54 \%)$ & \\
\hline \multicolumn{6}{|l|}{ Admission type } \\
\hline Emergency & $2728(90.96 \%)$ & $881(93.03 \%)$ & $937(89.32 \%)$ & $910(90.73 \%)$ & 0.016 \\
\hline Urgent & $179(5.97 \%)$ & $44(4.65 \%)$ & $80(7.63 \%)$ & $55(5.48 \%)$ & \\
\hline Elective & $92(3.07 \%)$ & $22(2.32 \%)$ & $32(3.05 \%)$ & $38(3.79 \%)$ & \\
\hline \multicolumn{6}{|l|}{ Comorbidity } \\
\hline Pulmonary circulatory disease & $255(8.50 \%)$ & $65(6.86 \%)$ & $88(8.39 \%)$ & $102(10.17 \%)$ & 0.032 \\
\hline Chronic pulmonary disease & $810(27.01 \%)$ & $223(23.55 \%)$ & $325(30.98 \%)$ & $262(26.12 \%)$ & $<0.001$ \\
\hline Renal failure & $356(11.87 \%)$ & $48(5.07 \%)$ & $121(11.53 \%)$ & $187(18.64 \%)$ & $<0.001$ \\
\hline Liver disease & $305(10.17 \%)$ & $34(3.59 \%)$ & $79(7.53 \%)$ & $192(19.14 \%)$ & $<0.001$ \\
\hline Metastatic cancer & $191(6.37 \%)$ & $44(4.65 \%)$ & $58(5.53 \%)$ & $89(8.87 \%)$ & $<0.001$ \\
\hline Congestive heart failure & $1064(35.48 \%)$ & $266(28.09 \%)$ & $396(37.75 \%)$ & $402(40.08 \%)$ & $<0.001$ \\
\hline SID30 & $11.31 \pm 8.32$ & $8.8 \pm 7.6$ & $10.9 \pm 8.1$ & $14.1 \pm 8.4$ & $<0.001$ \\
\hline \multicolumn{6}{|l|}{ Mechanical ventilation } \\
\hline Yes & $2649(88.33 \%)$ & $856(90.39 \%)$ & $940(89.61 \%)$ & $853(85.04 \%)$ & $<0.001$ \\
\hline No & $350(11.67 \%)$ & $91(9.61 \%)$ & $109(10.39 \%)$ & $150(14.96 \%)$ & \\
\hline \multicolumn{6}{|l|}{ Severity scale } \\
\hline APS III & $54.54 \pm 23.35$ & $48.94 \pm 21.70$ & $52.72 \pm 22.33$ & $61.74 \pm 24.10$ & $<0.001$ \\
\hline OASIS & $38.60 \pm 8.08$ & $37.77 \pm 7.69$ & $38.61 \pm 7.90$ & $39.36 \pm 8.56$ & $<0.001$ \\
\hline qSOFA & $1.89 \pm 0.68$ & $1.85 \pm 0.69$ & $1.92 \pm 0.67$ & $1.89 \pm 0.69$ & 0.113 \\
\hline SIRS & $3.14 \pm 0.89$ & $3.16 \pm 0.91$ & $3.14 \pm 0.87$ & $3.12 \pm 0.88$ & 0.501 \\
\hline \multicolumn{6}{|l|}{ Type of respiratory failure } \\
\hline Hypoxic respiratory failure & $2373(79.13 \%)$ & $762(80.46 \%)$ & $806(76.84 \%)$ & $805(80.26 \%)$ & 0.077 \\
\hline Hypercarbic respiratory failure & $626(20.87 \%)$ & $185(19.54 \%)$ & $243(23.16 \%)$ & $198(19.74 \%)$ & 0.062 \\
\hline Time in ICU (days) & $10.36 \pm 10.01$ & $10.43 \pm 10.61$ & $10.42 \pm 10.04$ & $10.24 \pm 9.38$ & 0.892 \\
\hline Time in hospital (days) & $17.10 \pm 16.37$ & $16.19 \pm 15.23$ & $17.21 \pm 15.63$ & $17.86 \pm 18.05$ & 0.076 \\
\hline 1-year mortality & $1443(48.12 \%)$ & $331(34.95 \%)$ & $454(43.28 \%)$ & $658(65.60 \%)$ & $<0.001$ \\
\hline 3-year mortality & $1654(55.15 \%)$ & $398(42.03 \%)$ & $530(50.52 \%)$ & $726(72.38 \%)$ & $<0.001$ \\
\hline
\end{tabular}

Table 1. Characteristics of the enrolled patients according to RDW. RDW red cell distribution width, SID30 Elixhauser Comorbidity Index, APS III Acute Physiology Score III, OASIS Oxford acute severity of illness score, $q S O F A$ quick sequential organ failure assessment score, SIRS systemic inflammatory response syndrome.

also had a high 1-year and 3-year mortality. The characteristics and hematologic laboratory data of the study participants were displayed in Table 1.

Association of RDW levels and clinical outcomes. The univariate Cox regression analysis (Table S2) indicated that age, gender, APS III score, OASIS score, qSOFA score, SIRS score, SID30, mechanical ventilation, congestive heart failure, chronic renal failure, liver disease, and metastatic cancer were significantly associated with 3-year all-cause mortality. The above variables also were associated with 1-year mortality (Table S3).

Figure 1 presents the association between baseline RDW levels and risk of 1-year and 3-year mortality (log $\mathrm{HR}$ ). Taking RDW on admission as a continuous variable, there was a significant positive association with the clinical outcomes in different multivariable models. We further evaluated this finding by the multivariable Cox regression analysis, which was shown in Table 2. As a continuous variable, an SD increase in RDW levels was associated with a $10 \%$ higher risk of 3 -year mortality in the adjusted model (HR 1.10, 95\% CI 1.07, 1.12, $\mathrm{P}<0.0001$ ), and there was a similar trend for 1 -year mortality (HR 1.10, 95\% CI 1.07, 1.12, $\mathrm{P}<0.0001)$. When RDW was assessed as tertiles, there was a significant high risk of 3-year mortality (HR 1.73, 95\% CI 1.52, 1.98, $\mathrm{P}<0.0001)$ and 1 -year mortality (HR 1.74, 95\% CI 1.51, 2.00, $\mathrm{P}<0.0001)$ in patients in high group compared with patients in low group.

Subgroup analyses were performed according to age, sex, ethnicity, pulmonary circulatory disease, chronic pulmonary disease, renal failure, liver disease, metastatic cancer, congestive heart failure and mechanical 
(A)

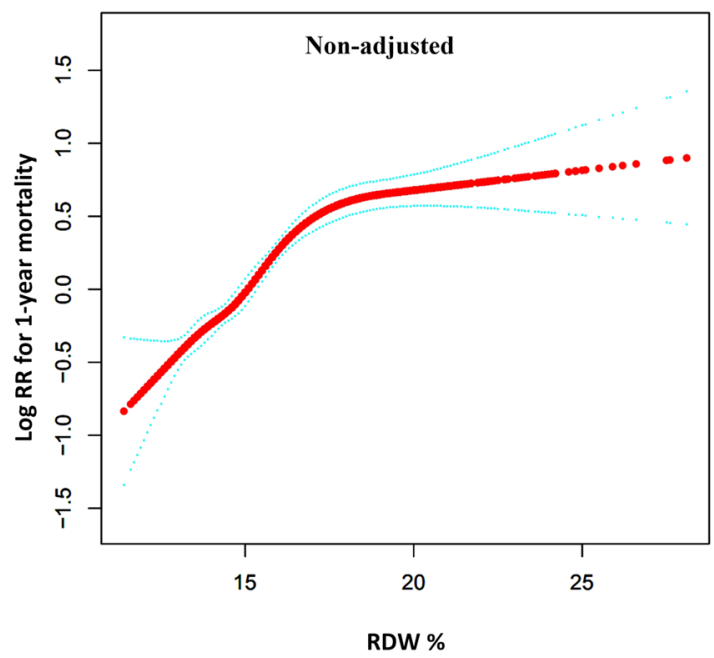

(C)

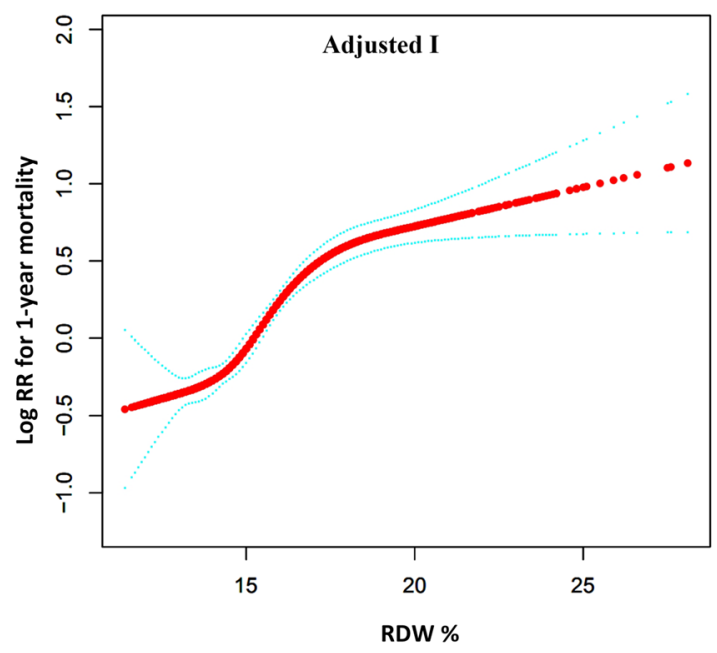

(E)

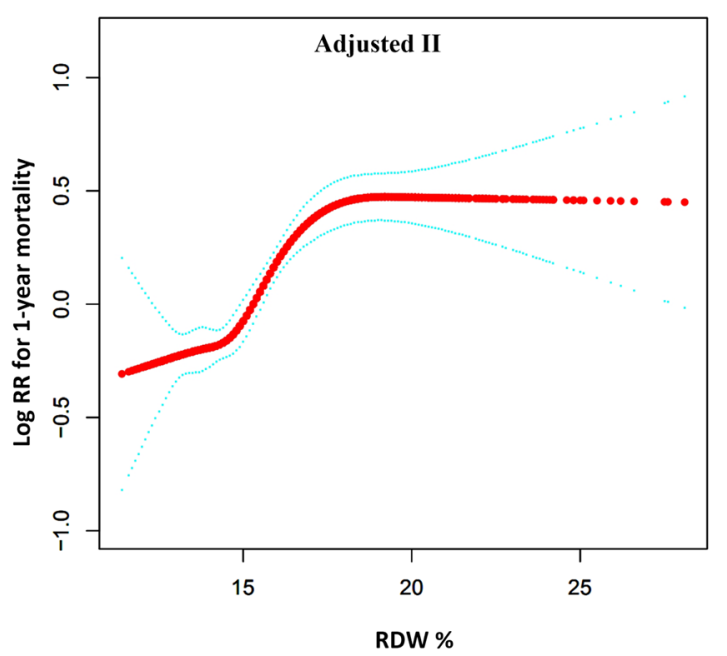

(B)

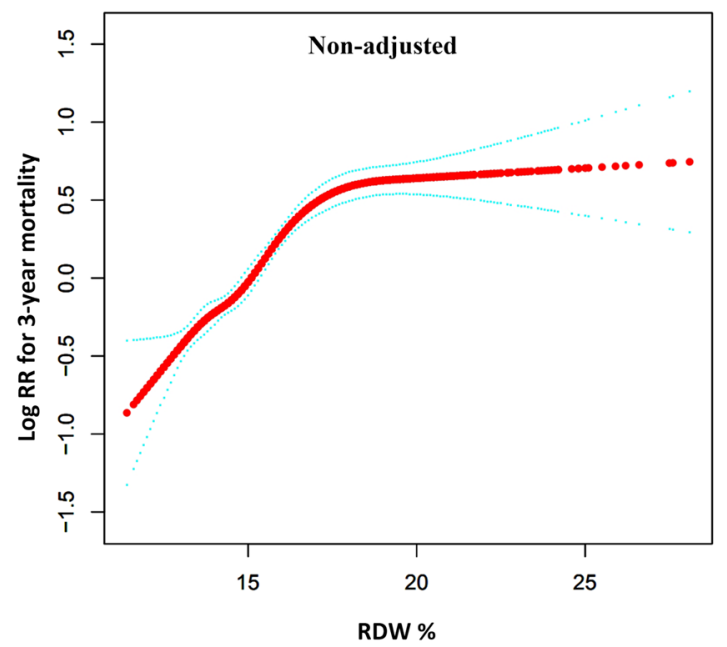

(D)

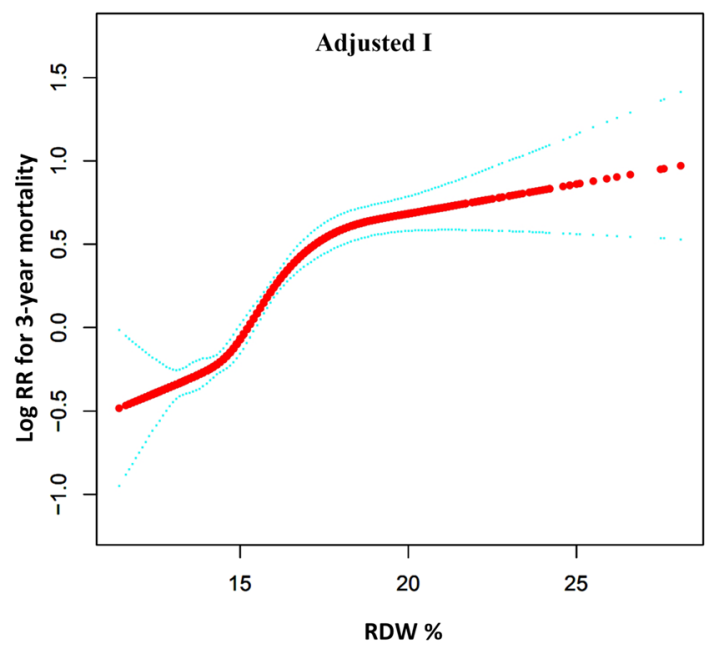

(F)

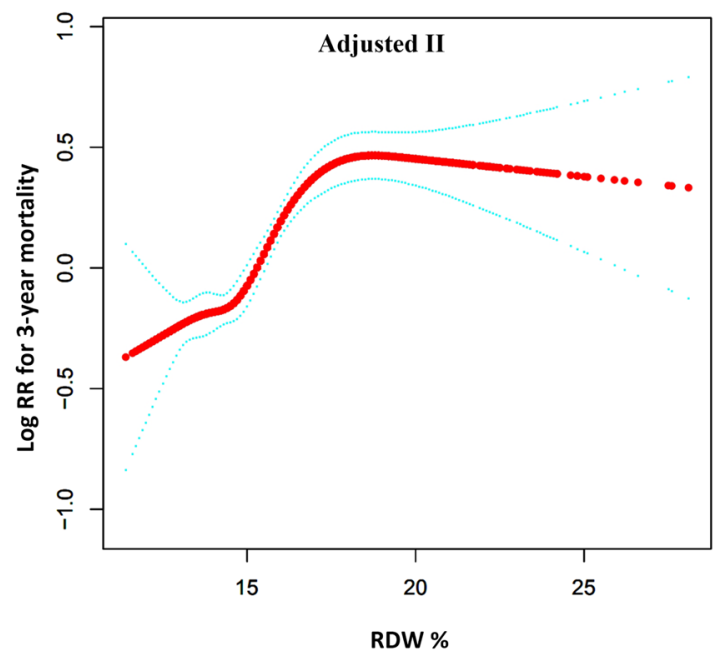

Figure 1. Association between RDW and clinical outcomes for patients with ARF in different multivariable models. $(\mathbf{A}, \mathbf{C}, \mathbf{E})$ association between RDW and 1-year mortality for patients with ARF. (B,D,F) association between RDW and 3-year mortality for patients with ARF. Adjusted I for age, gender and ethnicity; Adjusted II for age, gender, ethnicity, liver disease, metastatic cancer, congestive heart failure, renal failure, APS III score, SID30, OASIS, qSOFA, SIRS and mechanical ventilation. $H R$ indicates hazard risk, $A R F$ acute respiratory failure, $R D W$ red cell distribution width, SID30 Elixhauser Comorbidity Index, APS III acute physiology score III, OASIS Oxford acute severity of illness score, qSOFA quick sequential organ failure assessment score, SIRS Systemic inflammatory response syndrome. 


\begin{tabular}{|c|c|c|c|c|c|c|}
\hline \multirow[b]{2}{*}{ RDW, \% } & \multicolumn{2}{|l|}{ Non-adjusted } & \multicolumn{2}{|l|}{ Adjust I } & \multicolumn{2}{|l|}{ Adjust II } \\
\hline & HR (95\% CI) & P-value & HR (95\% CI) & P-value & HR $(95 \% \mathrm{CI})$ & P-value \\
\hline \multicolumn{7}{|c|}{ 1-year mortality } \\
\hline RDW Per 1 sd & $1.15(1.13,1.18)$ & $<0.0001$ & $1.16(1.14,1.18)$ & $<0.0001$ & $1.10(1.07,1.12)$ & $<0.0001$ \\
\hline \multicolumn{7}{|l|}{ RDW tertile } \\
\hline $\mathrm{T} 1$ & 1.0 (Ref) & & 1.0 (Ref) & & 1.0 (Ref) & \\
\hline $\mathrm{T} 2$ & $1.28(1.11,1.47)$ & 0.0006 & $1.19(1.03,1.37)$ & 0.0187 & $1.11(0.96,1.28)$ & 0.1534 \\
\hline $\mathrm{T} 3$ & $2.36(2.07,2.70)$ & $<0.0001$ & $2.22(1.95,2.54)$ & $<0.0001$ & $1.74(1.51,2.00)$ & $<0.0001$ \\
\hline \multicolumn{7}{|c|}{ 3-year mortality } \\
\hline RDW Per 1 sd & $1.15(1.13,1.17)$ & $<0.0001$ & $1.15(1.13,1.17)$ & $<0.0001$ & $1.10(1.07,1.12)$ & $<0.0001$ \\
\hline \multicolumn{7}{|l|}{ RDW tertile } \\
\hline $\mathrm{T} 1$ & 1.0 (Ref) & & 1.0 (Ref) & & 1.0 (Ref) & \\
\hline $\mathrm{T} 2$ & $1.26(1.11,1.44)$ & 0.0004 & $1.17(1.03,1.33)$ & 0.0194 & $1.11(0.97,1.26)$ & 0.1237 \\
\hline T3 & $2.30(2.03,2.60)$ & $<0.0001$ & $2.15(1.90,2.44)$ & $<0.0001$ & $1.73(1.52,1.98)$ & $<0.0001$ \\
\hline
\end{tabular}

Table 2. Association of RDW with 1-year mortality and 3-year mortality. Adjusted I for age, gender and ethnicity. Adjusted II for age, gender, ethnicity, liver disease, metastatic cancer, congestive heart failure, renal failure, APS III score, SID30, OASIS, qSOFA, SIRS and mechanical ventilation. HR indicates hazard risk, CI confidence interval.

ventilation for the primary and secondary outcomes. The association between RDW on admission and the clinical outcomes was similar for all strata (Tables 3, S4).

ROC curve analysis. ROC curves of 3-year mortality and 1-year mortality generated using the indicated variables (RDW, APS III, OASIS, qSOFA and SIRS) are plotted in Fig. 2A,B. The AUC of 3-year all-cause mortality for RDW was 0.651 (95\% CI $0.631,0.670)$, which was significantly higher than qSOFA and SIRS scores $(\mathrm{P}<0.0001$, Fig. 2B). And for 1-year mortality, the AUC was 0.652 (95\% CI 0.632, 0.671), which is also higher than qSOFA and SIRS scores. According to the $\mathrm{CO}_{2}$ levels in arterial blood, respiratory failure is divided into hypoxic respiratory failure and hypercarbic respiratory failure. We further analyzed the long-term predictive value of RDW for different types of respiratory failure, and the results were consistent (Fig. 2C-F). The AUC and $\mathrm{P}$ value of RDW and different scoring systems for clinical outcomes were shown in Table 4.

Survival status of patients with different admission RDW level. The K-M survival curves showed that patients in the high RDW group had significantly shorter survival time and higher mortality than patients in the middle and low RDW group (log-rank test: $\mathrm{P}<0.001$ ), which was presented in Fig. $3 \mathrm{~A}$. And for different types of respiratory failure, we obtain the same results (Fig. 3B,C).

\section{Discussion}

Our results demonstrated that there is a significant positive correlation between RDW on admission and longterm mortality of patients with ARF. High RDW was associated with high 3-year and 1-year mortality, and this correlation was also demonstrated in all subgroup analyses. We further analyzed the relationship between the number of reticulocyte and the RDW grouping, and the results indicated that the count of reticulocyte increased gradually according to the three groups (low, medium and high) of RDW patients, with a significant difference (Table S5). The results suggest a fact that hypoxemia lead to the increase of red blood cells, thus affecting red blood cell size.

RDW represents the size variation of circulating red blood cells. Any physiologic process that affects the morphology of red blood cells and causes the early release of young cells into circulation can lead to the increase of RDW. First, ARF can lead to severe hypoxemia which induces EPO release that can reach a level of several 100 -fold ${ }^{17}$. After the onset of ARF, hypoxia can lead to the production of hypoxia-inducible factors (HIFs) which may induced cell-type specific gene expression changes, thus, increasing the production of EPO in the renal and liver under the action of hypoxia-induced transcription factor 2 (HIF-2) ${ }^{17}$. EPO increases regardless of hypoxemia from a variety of causes, including COPD, pulmonary hypertension, interstitial lung disease, or heart failure ${ }^{18-20}$. The EPO not only increases the rate of formation of RBCs, but also increases the volume of RBCs leading to an increase in RDW ${ }^{18,21}$. Second, Previous studies have shown that RDW is associated with the outcomes of several inflammatory diseases, including acute pancreatitis, sepsis, and chronic heart failure ${ }^{22-25}$. Abnormal RDW elevation is closely related to proinflammatory factors ${ }^{24,26}$. Proinflammatory cytokines can decrease the half-life of red blood cells and change the morphology of red blood cells ${ }^{27}$. Moreover, inflammation can retard the maturation of red blood cells, causing an upregulation of reticulocytosis and the release of a large number of reticulocytes into the peripheral circulation, leading to the increase of $\mathrm{RDW}^{28,29}$. Third, in patients with mechanical ventilation and acute lung injury, oxidative stress presents to reactive active oxygen free radicals which can curtail the lifespan of red blood cells, thus promoting the release of young cells into the circulation ${ }^{30}$. Renal failures, hyperglycemia and vitamin D3 deficiency have also been suggested as factors linking RDW to poor clinical outcomes ${ }^{6}$. 


\begin{tabular}{|c|c|c|c|}
\hline & No. of patients & HR (95\% CI) & P-value \\
\hline \multicolumn{4}{|l|}{ Gender } \\
\hline Male & 1651 & $1.13(1.10,1.17)$ & $<0.0001$ \\
\hline Female & 1348 & $1.07(1.04,1.10)$ & $<0.0001$ \\
\hline \multicolumn{4}{|c|}{ Age (years) } \\
\hline$<65$ & 1433 & $1.13(1.10,1.17)$ & $<0.0001$ \\
\hline$\geq 65$ & 1566 & $1.07(1.04,1.10)$ & $<0.0001$ \\
\hline \multicolumn{4}{|l|}{ Ethnicity } \\
\hline Caucasian & 2072 & $1.10(1.07,1.13)$ & $<0.0001$ \\
\hline Black & 208 & $1.10(1.01,1.19)$ & 0.0202 \\
\hline Asian & 71 & $1.08(0.90,1.29)$ & 0.4193 \\
\hline Others & 648 & $1.08(1.03,1.13)$ & 0.0018 \\
\hline \multicolumn{4}{|c|}{ Congestive heart failure } \\
\hline No & 1935 & $1.09(1.06,1.12)$ & $<0.0001$ \\
\hline Yes & 1064 & $1.10(1.07,1.14)$ & $<0.0001$ \\
\hline \multicolumn{4}{|c|}{ Pulmonary circulatory disease } \\
\hline No & 2744 & $1.10(1.07,1.12)$ & $<0.0001$ \\
\hline Yes & 255 & $1.10(1.02,1.18)$ & 0.0092 \\
\hline \multicolumn{4}{|c|}{ Chronic pulmonary disease } \\
\hline No & 2189 & $1.09(1.06,1.12)$ & $<0.0001$ \\
\hline Yes & 810 & $1.11(1.06,1.16)$ & $<0.0001$ \\
\hline \multicolumn{4}{|c|}{ Renal failure } \\
\hline No & 2643 & $1.09(1.06,1.11)$ & $<0.0001$ \\
\hline Yes & 356 & $1.17(1.10,1.24)$ & $<0.0001$ \\
\hline \multicolumn{4}{|c|}{ Liver disease } \\
\hline No & 2694 & $1.10(1.07,1.12)$ & $<0.0001$ \\
\hline Yes & 305 & $1.06(1.01,1.12)$ & 0.0262 \\
\hline \multicolumn{4}{|c|}{ Metastatic cancer } \\
\hline No & 2808 & $1.10(1.08,1.13)$ & $<0.0001$ \\
\hline Yes & 191 & $1.04(0.98,1.11)$ & 0.2327 \\
\hline \multicolumn{4}{|c|}{ Mechanical ventilation } \\
\hline No & 350 & $1.14(1.08,1.21)$ & $<0.0001$ \\
\hline Yes & 2649 & $1.09(1.06,1.11)$ & $<0.0001$ \\
\hline
\end{tabular}

Table 3. Subgroup analysis of the associations between RDW and 3-year all-cause mortality by multivariable Cox regression. Adjusted for age, gender, ethnicity, liver disease, metastatic cancer, congestive heart failure, renal failure, APS III score, SID30, OASIS, qSOFA, SIRS and mechanical ventilation, if not stratified. $H R$ indicates hazard risk, $C I$ confidence interval.

Combined with the results of reticulocyte and previous studies, we proposed the following hypothesis: after the onset of ARF, hypoxemia can promote the synthesis of EPO in the kidney and liver, thereby increasing the rate of formation of RBCs and affecting the morphology of red blood cells. Inflammation and oxidative stress prevent the maturation of red blood cells, resulting in the release of large numbers of reticulocytes into the peripheral circulation and leading to the increase of RDW.

To our knowledge, this is the first study to demonstrate high RDW as an independent predictor for the longterm outcomes of patients with ARF. Previous studies have shown that high RDW is associated with a lower $\mathrm{PaO} 2 / \mathrm{FiO} 2$ rate, a lower ventilation-free day and a greater need for ventilation in acute respiratory failure. Tiffany et al. ${ }^{28}$ found that in a cohort of 637 patients with ARF, participants with high RDW at admission had a $32 \%$ reduction in total length of 30-day period without ventilator compared with the patients with RDW in normal range (RR: $0.68 ; 95 \%$ CI 0.55-0.83, $\mathrm{P}<0.001$ ). Tom et al. ${ }^{14}$ showed that after adjusting for anemia, age, and disease severity (PRISM III), high RDW was an independent risk factor for mechanical ventilation of children (OR 2.6; 95\% CI 1.4-4.9; $\mathrm{P}=0.004)$. Danny et al. ${ }^{31}$ constructed survival curves after adjusting for Charlson comorbidity index and reported that 60-day readmission for AECOPD was higher in patients with high RDW than patients with normal RDW $(\mathrm{P}=0.0038)$. Moreover, results from a retrospective cohort study of MIMIC-III data including 404 eligible ARDS patients suggest that RDW $\geq 14.5 \%$ was an independent predictor of 30 -day (OR 1.91, 95\% CI 1.08, 3.39) and 90-day mortality (OR 2.56, 95\% CI 1.50, 4.37) ${ }^{13}$. These findings are similar to our results and high RDW is associated with poor prognosis in patients with respiratory failure.

In addition, we compared the value of RDW and other severity scale scores to predict long-term mortality. The results showed that, the AUC of RDW has a significant higher predictive value than the qSOFA and SIRS scores. In our study, only APS III had predictive accuracy comparable to RDW on long-term clinical outcomes for 
(A)

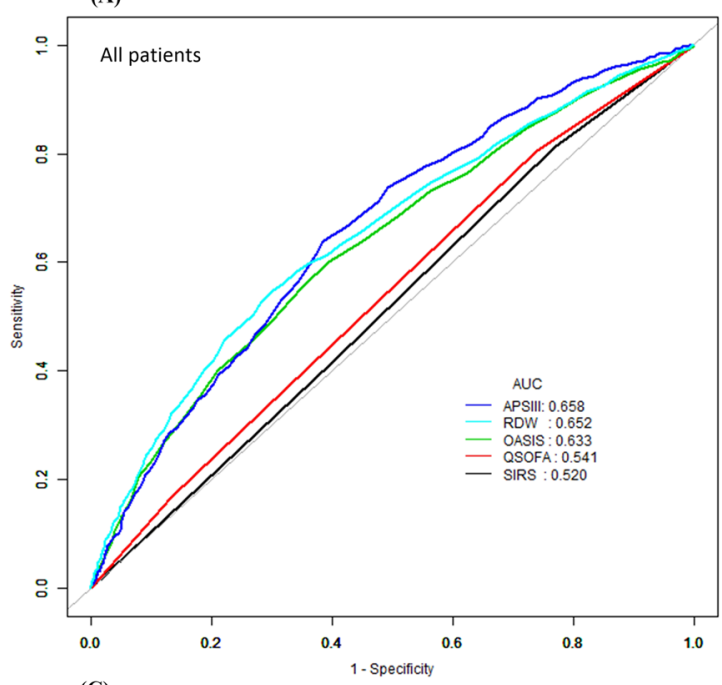

(C)

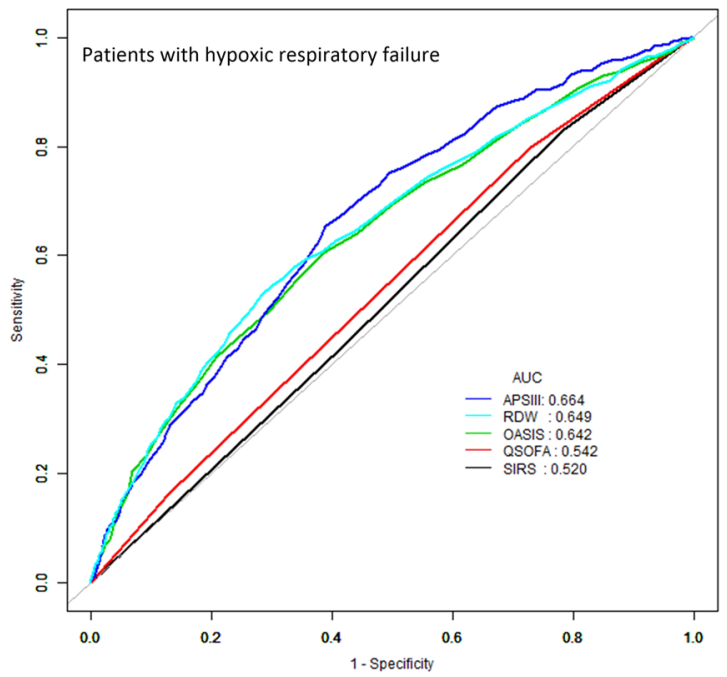

(E)

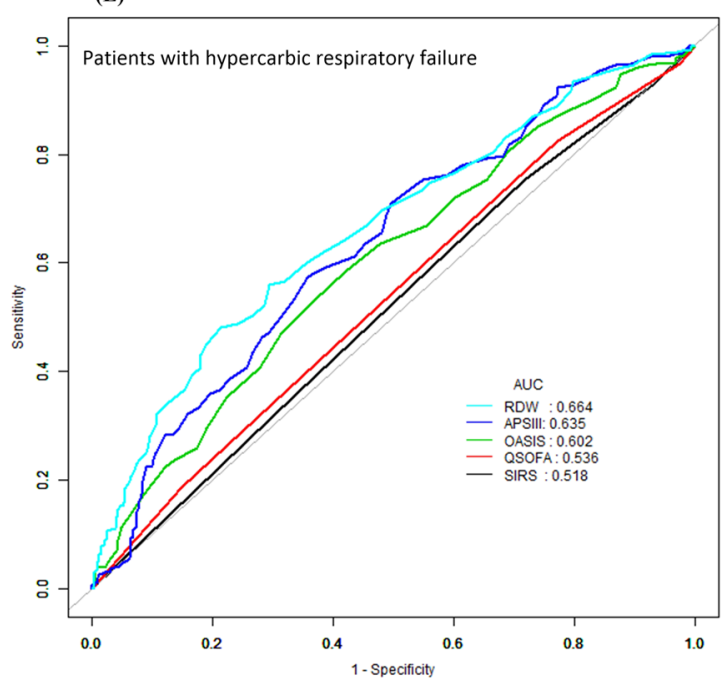

(B)

ROC of 3-year mortality

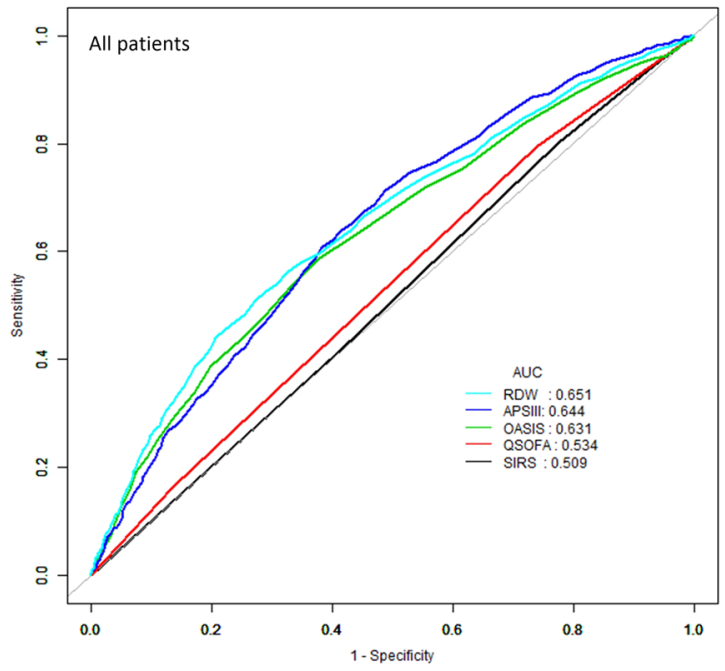

(D)

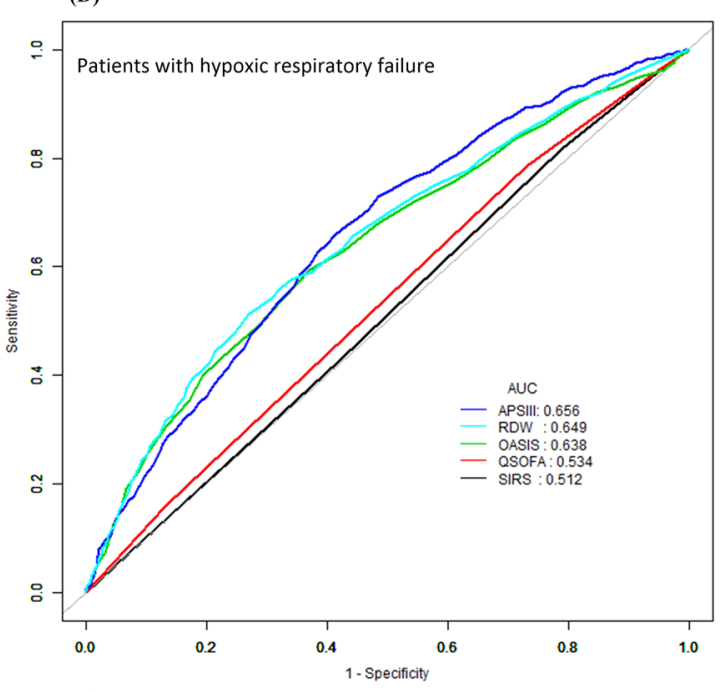

(F)

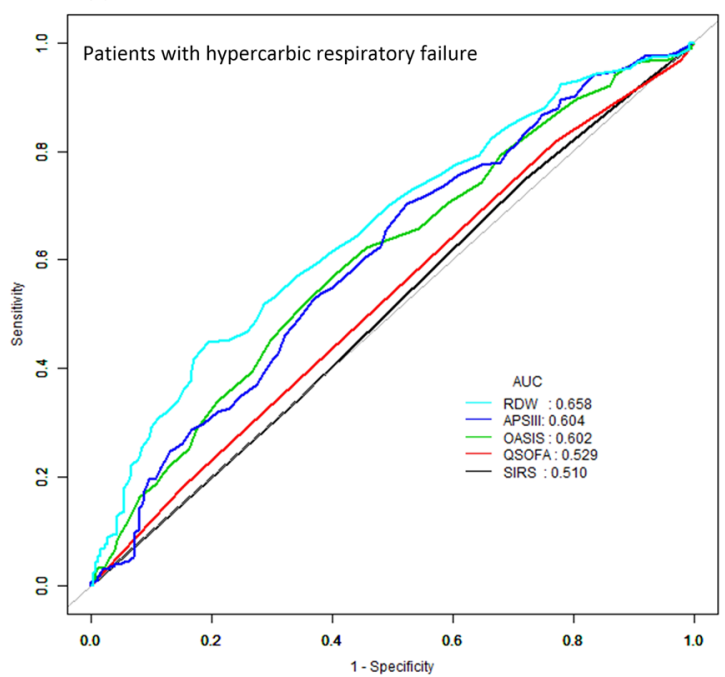

Figure 2. ROC analyses of predictors of red cell distribution width (RDW) for 3-year and 1-year mortality in critical ill patients, which were compared with APS III, OASIS, qSOFA and SIRS. (A,B) ROC for all patients; (C,D) ROC for patients with hypoxic respiratory failure; (E,F) ROC for patients with hypercarbic respiratory failure. APS III acute physiology score III, OASIS Oxford acute severity of illness score, qSOFA quick sequential organ failure assessment score, SIRS Systemic inflammatory response syndrome, $R O C$ receiver operating curve. 


\begin{tabular}{|c|c|c|c|c|c|c|}
\hline \multirow[b]{2}{*}{ Predictor } & \multicolumn{2}{|c|}{ All patients } & \multicolumn{2}{|c|}{$\begin{array}{l}\text { Patients } \\
\text { with hypoxic } \\
\text { respiratory } \\
\text { failure }\end{array}$} & \multicolumn{2}{|c|}{$\begin{array}{l}\text { Patients with } \\
\text { hypercarbic } \\
\text { respiratory } \\
\text { failure }\end{array}$} \\
\hline & AUC & P-value & AUC & P-value & AUC & P-value \\
\hline \multicolumn{7}{|c|}{ 1-year mortality } \\
\hline RDW & 0.652 & & 0.649 & & 0.664 & \\
\hline APS III & 0.658 & 0.6475 & 0.664 & 0.2748 & 0.635 & 0.3214 \\
\hline OASIS & 0.633 & 0.1815 & 0.642 & 0.6393 & 0.602 & 0.0521 \\
\hline qSOFA & 0.541 & $<0.0001$ & 0.542 & $<0.0001$ & 0.536 & $<0.0001$ \\
\hline SIRS & 0.520 & $<0.0001$ & 0.520 & $<0.0001$ & 0.518 & $<0.0001$ \\
\hline \multicolumn{7}{|c|}{ 3-year mortality } \\
\hline RDW & 0.651 & & 0.649 & & 0.658 & \\
\hline APS III & 0.644 & 0.6267 & 0.656 & 0.6060 & 0.604 & 0.0811 \\
\hline OASIS & 0.631 & 0.1495 & 0.638 & 0.4867 & 0.602 & 0.0848 \\
\hline qSOFA & 0.534 & $<0.0001$ & 0.534 & $<0.0001$ & 0.529 & $<0.0001$ \\
\hline SIRS & 0.509 & $<0.0001$ & 0.512 & $<0.0001$ & 0.510 & $<0.0001$ \\
\hline
\end{tabular}

Table 4. ROC of RDW and severity scales for different types of respiratory failure. $R O C$ receiver operating curve, $A U C$ area under curve.
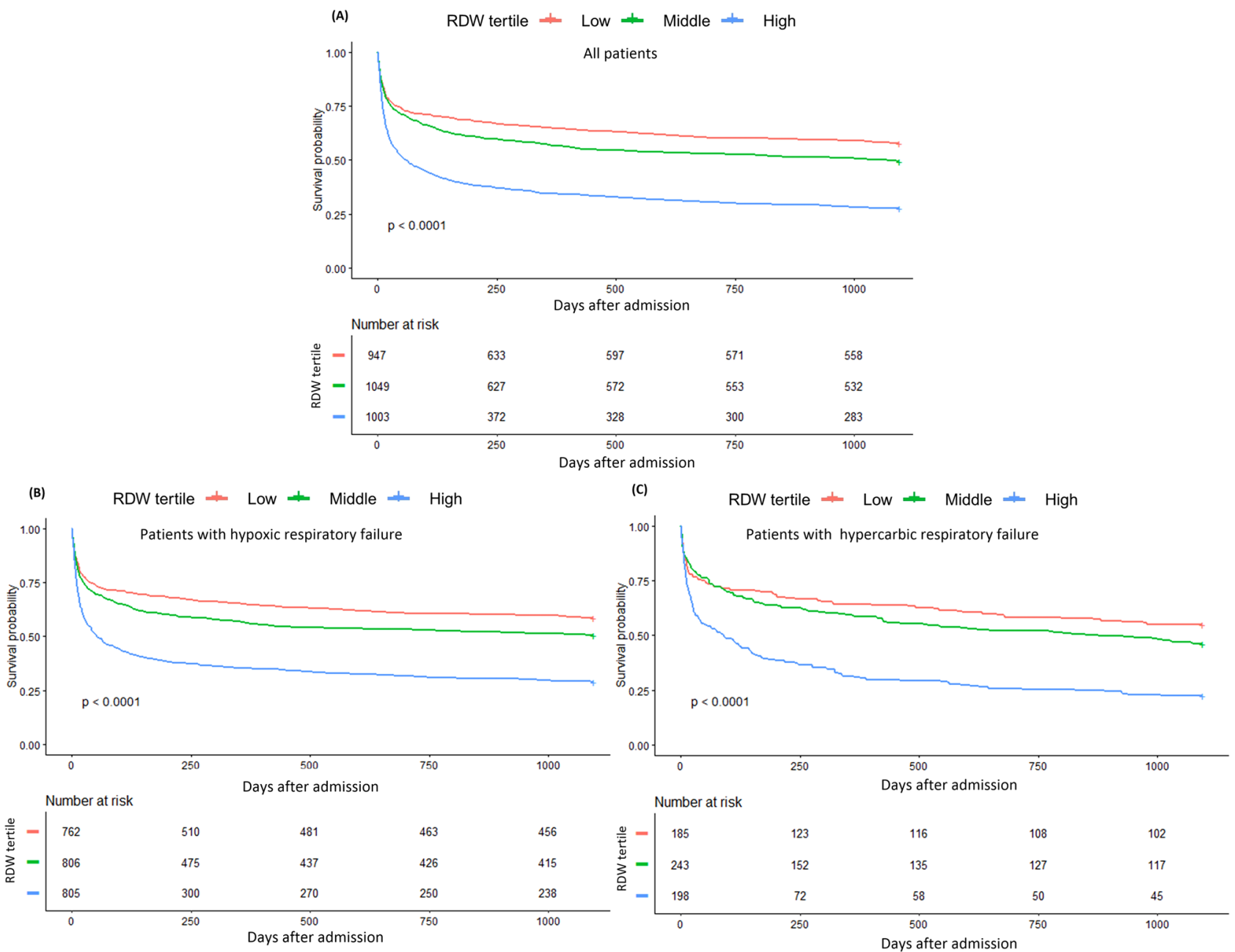

Figure 3. Kaplan-Meier (K-M) survival curves for 3-year all-cause mortalities. (A) 3-year all-cause mortalities for all patients; (B) 3-year all-cause mortalities for patients with hypoxic respiratory failure; (C) 3-year all-cause mortalities for patients with hypercarbic respiratory failure. 
ARF. However, the clinical application of APS III is very limited, which requires more patients' data to calculate, including vital signs, blood gas analysis, laboratory test results, etc. On the contrary, RDW is a routine parameter of blood routine. It can be obtained easily without any additional cost, which may make it more convenient for clinical use to early detect the patients with poor long-term prognosis, and early to take different treatment and nursing measures.

The advantage of the present study is that we analyzed the long-term prognosis of ARF which is difficult to be observed in other studies, because we had a complete follow-up database and a large sample size. However, our study has some limitations. First, as a retrospective cohort study, it is impossible to adjust for all confounders. We have adjusted for known confounders as much as possible, but there are still some unmeasured variables that may affect our results. Due to several variables were not recorded in MIMIC-III, we lacked some indicators about inflammatory response (such as C-reactive protein and interleukin 6) which may affect our results. Therefore, prospective studies are needed to confirm these results. Second, we only measured RDW levels of patients on admission; we did not investigate the trends, which could reveal more information. Third, this is a single-center study, and the results of the study should also be interpreted with caution when implicating in other populations and areas. Fourth, considering the effects of blood transfusion and anemia on RDW, patients with anemia and received blood transfusion were excluded according to the ICD9 code of MIMIC database. Therefore, our conclusions could not interpret these patients.

\section{Conclusion}

In conclusion, our data suggested an association between the RDW and survival time of 3-year follow-up, particularly a high RDW on admission was associated with an increased risk of long-term mortality in patients with ARF. RDW may provide an alternative indicator to predict the prognosis and disease progression and more it is easy to get. There are several theoretical mechanisms to explain the increase of RDW, and further studies are needed to validate the relationship between EPO, pro-inflammatory factors, reticulocytes, and RDW in the serum of patients with ARF.

\section{Patients and methods}

Data sources. This is a retrospective cohort study with the data of patients obtained from the Medical Information Mart for Intensive Care III (MIMIC-III). We gained access to the database by taking an online course at the National Institutes of Health and passing the 'protection Human Research Participants' exam (no. 6182750). MIMIC III is a single-center and freely accessible database which contains 53,423 adult patients (aged over 16) during 2001 to 2012 in the ICU of the Beth Israel Deaconess Medical Center in Boston ${ }^{32}$. The establishment of the MIMIC III database was approved by the institutional review board of Beth Israel deacons Medical Center and Massachusetts Institute of Technology. Informed consent was not required because all protected health information has been de-identified.

Patients. All the patients who were over the age of 18 and hospitalized in ICU for more than $48 \mathrm{~h}$ were included in the study. Patients who did not have an RDW data within $24 \mathrm{~h}$ after admission were excluded. Patients with anemia or received blood transfusion were also excluded. If a patient was admitted repeatedly during the study period, we used only the record of his first hospital admission (Fig. 4).

Variables and outcome measures. The following variables were extracted or calculated: age, sex, comorbidities, ethnicity, admission type, 1-year and 3-year mortality, length of ICU and hospital stay, RDW (within 24hour of ICU admission), mechanical ventilation, Acute Physiology Score III (APS III), the Oxford Acute Severity of Illness Score (OASIS), the quick Sequential Organ Failure Assessment score (qSOFA), the Systemic Inflammatory Response Syndrome score (SIRS) and the Elixhauser Comorbidity Index (SID30). The APS III, OASIS, qSOFA and SIRS scores were estimated for all patients within $24 \mathrm{~h}$ of ICU admission. The comorbidities were identified and extracted by ICD-9 code in MIMIC-III database. Detailed ICD-9 codes and disease names of each comorbidities are presented in S1. The codes of Structured Query Language which was used to extract the data were obtained from https://github.com/MIT-LCP/mimic-website 32 . The primary outcome was the 3-year mortality following hospital admission, and the secondary outcome was 1-year mortality.

Statistical analysis. The whole participants were divided into three groups according to the tertiles of RDW. The categorical variables were presented as percentages and the continuous variables were expressed as the mean (SD) or IQR. The baseline characteristics and the scoring systems were compared by chi-square test when they were categorical variables and by Kruskal-Wallis test when they were continuous variables for the three groups. Multivariable Cox regression model and generalized linear models with a logit link were used to test the independent effects of RDW on 3-year and 1-year all-cause mortality with crude and full model. The adjusting variables included age, gender, ethnicity, liver disease, metastatic cancer, congestive heart failure, renal failure, APS III score, ECI (SID30), OASIS, qSOFA, SIRS and mechanical ventilation. We selected those confounders based on their associations with the outcomes of interest or a change in effect estimate of more than $10 \%$.

Additionally, a subgroup analysis was performed to determine whether there were differences of each subgroup in RDW prediction of clinical outcomes. The effects of RDW (as a continuous variable) on the risks of ARF were estimated using COX regression models among subgroups classified by adjusting for age, gender, ethnicity, liver disease, metastatic cancer, congestive heart failure, renal failure, APS III score, SID30, OASIS, qSOFA, SIRS and mechanical ventilation, if not stratified. The Kaplan-Meier (K-M) method and log-rank tests were used to compare the differences in survival rate between each group of patients with RDW at admission. 
53423 in-patients were identified from MIMIC-III
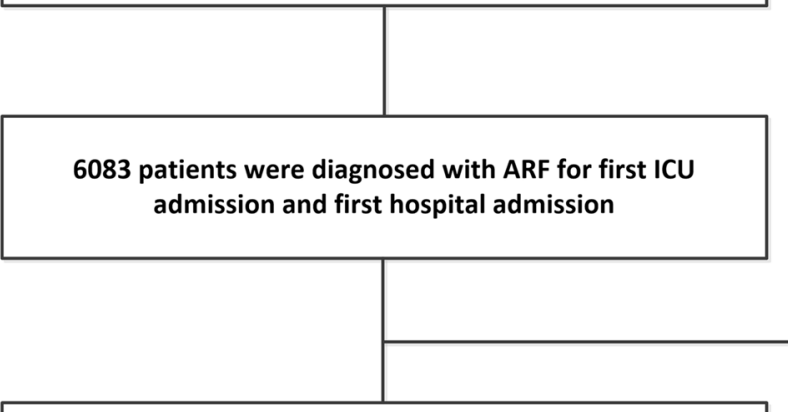

1223 patients were hospitalized for less than $\mathbf{4 8}$ hours 24 patients aged under 18 years old

4836 patients with ARF were selected

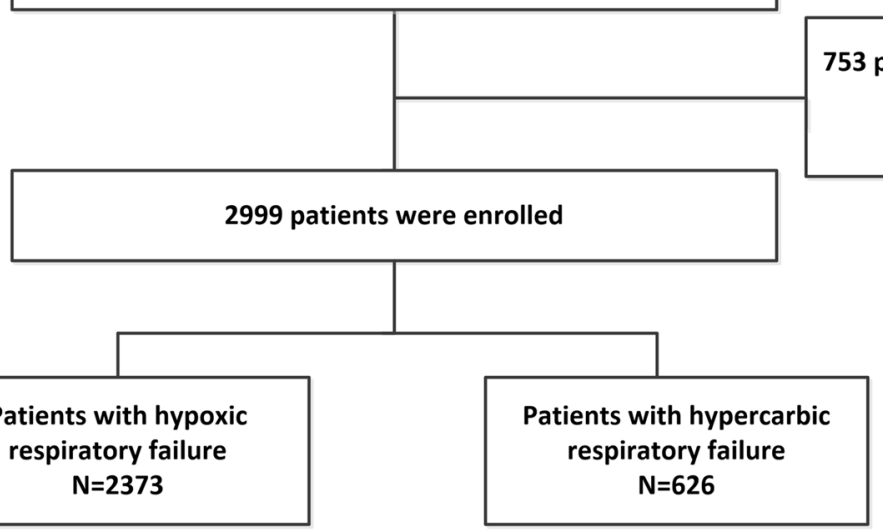

753 patients lacked RDW data within 24 hours after admission 1033 patients were diagnosed with anemia 51 patients received blood transfusions

Figure 4. Flow chart of the current study. $A R F$ acute respiratory failure, $R D W$ red cell distribution width, $I C U$ intensive care unit.

We performed the Receiver operating characteristic (ROC) curves to assess the predictive value of RDW for 3 -year and 1-year mortality of patients.

All the data were processed and analyzed by EmpowerStats software (www.empowerstats.com version R.3.4.3) and statistical software package $\mathrm{R}$. A two-tailed $\mathrm{P}$ value $<0.05$ was considered statistically significant.

Received: 3 June 2020; Accepted: 20 November 2020

Published online: 03 December 2020

\section{References}

1. Scala, R. \& Heunks, L. Highlights in acute respiratory failure. Eur. Respir. Rev. 27, 180008 (2018).

2. Lamba, T. S., Sharara, R. S., Singh, A. C. \& Balaan, M. Pathophysiology and classification of respiratory failure. Crit. Care Nurs. Q. 39, 85-93 (2016).

3. Kumar, S., Khilnani, G. C., Banga, A. \& Sharma, S. K. Predictors of requirement of mechanical ventilation in patients with chronic obstructive pulmonary disease with acute respiratory failure. Lung India 30, 178-182 (2013).

4. Ocal, S. et al. Long-term outcome of chronic obstructive pulmonary disease patients with acute respiratory failure following intensive care unit discharge in Turkey. Clin. Respir. J. 11, 975-982 (2017).

5. Dziadzko, M. A. et al. Multicenter derivation and validation of an early warning score for acute respiratory failure or death in the hospital. Crit. Care 22, 286 (2018).

6. Salvagno, G. L., Sanchis-Gomar, F., Picanza, A. \& Lippi, G. Red blood cell distribution width: a simple parameter with multiple clinical applications. Crit. Rev. Clin. Lab. Sci. 52, 86-105 (2015).

7. Lorente, L. et al. Early mortality of brain infarction patients and red blood cell distribution width. Brain Sci. 10, 196 (2020).

8. Dai, H., Su, X., Li, H. \& Zhu, L. Association between red blood cell distribution width and mortality in diabetic ketoacidosis. J. Int. Med. Res. 48, 1220711046 (2020).

9. Wei, S. et al. Red blood cell distribution width predicts postoperative death of infective endocarditis. Int. Heart J. 61, 524 (2020).

10. Hu, Z. D., Lippi, G. \& Montagnana, M. Diagnostic and prognostic value of red blood cell distribution width in sepsis: a narrative review. Clin. Biochem. 77, 1-6 (2020).

11. Wang, L. et al. Red blood cell distribution width is associated with mortality after acute ischemic stroke: a cohort study and systematic review. Ann. Transl. Med. 8, 81 (2020).

12. Lochowski, M. et al. Prognostic value of red blood cell distribution width-standard deviation (RDW-SD) in patients operated on due to non-small cell lung cancer. J. Thorac. Dis. 12, 773-781 (2020).

13. Wang, B., Gong, Y., Ying, B. \& Cheng, B. Relation between red cell distribution width and mortality in critically ill patients with acute respiratory distress syndrome. Biomed. Res. Int. 2019, 1-8 (2019). 
14. Schepens, T., De Dooy, J. J., Verbrugghe, W. \& Jorens, P. G. Red cell distribution width (RDW) as a biomarker for respiratory failure in a pediatric ICU. J. Inflamm. 14, 12 (2017).

15. Rahimirad, S., Ghafari, M., Ansarin, K., Rashidi, F. \& Rahimi-Rad, M. H. Elevated red blood cell distribution width predicts mortality in acute exacerbation of COPD. Pneumologia 65, 85-89 (2016).

16. $\mathrm{Hu}, \mathrm{G}$. P. et al. Red blood cell distribution width is an independent predictor of mortality for an acute exacerbation of COPD. Int. J. Tuberc. Lung Dis. 23, 817-823 (2019).

17. Haase, V. H. Regulation of erythropoiesis by hypoxia-inducible factors. Blood Rev. 27, 41-53 (2013).

18. Ycas, J. W., Horrow, J. C. \& Horne, B. D. Persistent increase in red cell size distribution width after acute diseases: a biomarker of hypoxemia?. Clin. Chim. Acta 448, 107-117 (2015).

19. Nagai, T. et al. Prognostic significance of endogenous erythropoietin in long-term outcome of patients with acute decompensated heart failure. Eur. J. Heart Fail. 18, 803-813 (2016).

20. Markoulaki, D. et al. Hemoglobin, erythropoietin and systemic inflammation in exacerbations of chronic obstructive pulmonary disease. Eur. J. Intern. Med. 22, 103-107 (2011).

21. Parisotto, R. et al. A novel method utilising markers of altered erythropoiesis for the detection of recombinant human erythropoietin abuse in athletes. Haematologica 85, 564-572 (2000).

22. Danese, E., Lippi, G. \& Montagnana, M. Red blood cell distribution width and cardiovascular diseases. J. Thorac. Dis. 7, E402-E411 (2015).

23. Inuzuka, R. \& Abe, J. Red blood cell distribution width as a link between ineffective erythropoiesis and chronic inflammation in heart failure. Circ. J. 79, 974-975 (2015).

24. Zhang, F. X., Li, Z. L., Zhang, Z. D. \& Ma, X. C. Prognostic value of red blood cell distribution width for severe acute pancreatitis. World J. Gastroenterol. 25, 4739-4748 (2019).

25. Han, Y. Q. et al. Red blood cell distribution width predicts long-term outcomes in sepsis patients admitted to the intensive care unit. Clin. Chim. Acta 487, 112-116 (2018).

26. Pierce, C. N. \& Larson, D. F. Inflammatory cytokine inhibition of erythropoiesis in patients implanted with a mechanical circulatory assist device. Perfusion 20, 83-90 (2005).

27. Wang, F. et al. Red cell distribution width as a novel predictor of mortality in ICU patients. Ann. Med. 43, 40-46 (2011).

28. Otero, T. et al. Elevated red cell distribution width is associated with decreased ventilator-free days in critically ill patients. J. Intens. Care Med. 33, 241-247 (2018).

29. Sadaka, F., O’Brien, J. \& Prakash, S. Red cell distribution width and outcome in patients with septic shock. J. Intens. Care Med. 28, 307-313 (2013).

30. Ycas, J. W. Toward a blood-borne biomarker of chronic hypoxemia: red cell distribution width and respiratory disease. Adv. Clin. Chem. 82, 105-197 (2017).

31. Epstein, D., Nasser, R., Mashiach, T., Azzam, Z. S. \& Berger, G. Increased red cell distribution width: a novel predictor of adverse outcome in patients hospitalized due to acute exacerbation of chronic obstructive pulmonary disease. Respir. Med. 136, 1-7 (2018).

32. Johnson, A. E. et al. MIMIC-III, a freely accessible critical care database. Sci. Data 3, 160035 (2016).

\title{
Acknowledgements
}

The authors thank all the researchers who created and managed the MIMIC III database. This study was funded by General Projects of Social Development in Shaanxi Province (2019SF-151).

\section{Author contributions}

W.Z. designed the study, collected and analyzed data, and contributed to writing this manuscript. Y.W. collected and analyzed data. J.W. and S.W. designed and supervised the study and drafted the manuscript. All authors have read and approved the final manuscript.

\section{Competing interests}

The authors declare no competing interests.

\section{Additional information}

Supplementary Information The online version contains supplementary material available at https://doi. org/10.1038/s41598-020-78321-2.

Correspondence and requests for materials should be addressed to W.Z.

Reprints and permissions information is available at www.nature.com/reprints.

Publisher's note Springer Nature remains neutral with regard to jurisdictional claims in published maps and institutional affiliations.

\begin{abstract}
Open Access This article is licensed under a Creative Commons Attribution 4.0 International License, which permits use, sharing, adaptation, distribution and reproduction in any medium or format, as long as you give appropriate credit to the original author(s) and the source, provide a link to the Creative Commons licence, and indicate if changes were made. The images or other third party material in this article are included in the article's Creative Commons licence, unless indicated otherwise in a credit line to the material. If material is not included in the article's Creative Commons licence and your intended use is not permitted by statutory regulation or exceeds the permitted use, you will need to obtain permission directly from the copyright holder. To view a copy of this licence, visit http://creativecommons.org/licenses/by/4.0/.
\end{abstract}

(C) The Author(s) 2020 